\title{
Complexification of the projective and injective tensor products
}

by

\author{
GUSTI VAN ZYL (Pretoria)
}

\begin{abstract}
We show that the Taylor (resp. Bochnak) complexification of the injective (projective) tensor product of any two real Banach spaces is isometrically isomorphic to the injective (projective) tensor product of the Taylor (Bochnak) complexifications of the two spaces.
\end{abstract}

1. Introduction. In the Résumé de la théorie métrique des produits tensoriels topologiques, Grothendieck writes the following: "Dans la théorie des $\otimes$-normes développés dans ce travail, il faut bien faire attention que le corps des scalaires $\mathbb{R}$ ou $\mathbb{C}$ est fixé une fois pour toutes. Il faut donc distinguer entre l'ensemble $\mathcal{C}_{r}$ des ' $\otimes$-normes réelles' et l'ensemble $\mathcal{C}_{c}$ des ' $\otimes$-normes complexes'. Les relations entre les deux ne semblent pas si simples qu'on pourrait s'y attendre" [3, p. 18].

One way to study relationships between real and complex tensor norms is through "complexification".

Most of the well-known Banach spaces have complex as well as real versions. For example, one has $\mathbb{C}^{n}$ as well as $\mathbb{R}^{n}$, and in function spaces one almost always finds an analogous Banach space of complex-valued functions whenever one has a Banach space of real-valued functions. In [4] the complexification of vector spaces is given a general axiomatic approach, and then the problem of the complexification of Banach spaces, i.e. finding the norm functional on the complex space from the norm functional on the real space, is considered.

A concrete description of their definition is to identify the complexification of a real vector space $X$ with $X \oplus X$, making the latter a complex vector space by defining scalar multiplication with the formula $(\alpha+i \beta)(x, y):=$ $(\alpha x-\beta y, \alpha y+\beta x)$, whenever $\alpha+i \beta \in \mathbb{C},(x, y) \in X \oplus X$.

2000 Mathematics Subject Classification: Primary 46B28.

Key words and phrases: complexification, projective tensor product, injective tensor product. 
It is natural to write $x+i y$ for $(x, y) \in X \oplus X$. The authors of [4] show which complexification procedure, that is, a method of assigning a norm to the complexification of the Banach space in a way that is applicable to all Banach spaces, or, in other words, defining it in a way that is not tied to the definition of any particular Banach space, is the smallest or largest; which procedure produces $\ell_{\mathbb{C}}^{1}$ from $\ell_{\mathbb{R}}^{1}$, and so on. One might also ask which properties of the Banach space are preserved by which complexification procedures.

In this article we consider the complexification of the injective and projective tensor products of real Banach spaces, and show in each case a complexification procedure that turns the injective (projective) tensor product of two Banach spaces into the injective (projective) tensor product of their complexifications.

As in, for example, [2], we denote the tensor product of Banach spaces $X$ and $Y$, endowed with the tensor norm $\alpha$, by $X \otimes_{\alpha} Y$, and the completion under this norm by $X \stackrel{\alpha}{\otimes} Y$. In this article, excepting the concluding remarks, $\alpha$ will be either the injective tensor norm $\alpha=\vee$ or the projective tensor norm $\alpha=\wedge$. We will use the symbols $\stackrel{1}{=}$ for isometric isomorphism, $C(K)$ for the Banach space of real-valued functions on the the compact Hausdorff space $K$, and $C_{\mathbb{C}}(K)$ for the Banach space of complex-valued functions on $K$.

2. The injective tensor product. For any real Banach space $X$, we write $X_{T}$ for its Taylor complexification, which means that the complexification $X \oplus X$ is endowed with the Taylor complexification norm,

$$
\|x+i y\|_{X_{T}}:=\sup _{0 \leq \theta \leq 2 \pi}\|(\cos \theta) x-(\sin \theta) y\|_{X} .
$$

(See $[4$, p. 6] for a discussion.) As the Taylor complexification of a $C(K)$ space is also the complexification obtained by the functional calculus approach on Banach lattices, as discussed in $\left[4\right.$, p. 6], which yields $C_{\mathbb{C}}(K)$, we have $C_{\mathbb{C}}(K) \stackrel{1}{=} C(K)_{T}$.

Before the main theorem of this section, we make a quick observation.

TheOREM 2.1. There is a linear bijection between the complex vector spaces $X_{T} \otimes_{\vee} Y_{T}$ and $\left(X \otimes_{\vee} Y\right)_{T}$.

Proof. Both spaces are the complex span of $\{x \otimes y: x \in X, y \in Y\}$.

Theorem 2.2. Let $u \in X_{T} \otimes_{\vee} Y_{T}$. Then $\|u\|_{X_{T} \otimes_{\vee} Y_{T}}=\|u\|_{\left(X \otimes_{\vee} Y\right)_{T}}$.

Proof. On the one hand, defining $Z_{X}:=\{\widehat{x}(\cdot) \mid x \in X\}$ as the image of the usual isometric imbedding of $X$ into a subspace of $C\left(B_{X^{*}}\right)$, and defining $Z_{Y}$ similarly, we have $X \stackrel{1}{=} Z_{X}$ and $Y \stackrel{1}{=} Z_{Y}$. 
By injectivity of $\otimes_{\mathrm{V}}$, we find $Z_{X} \otimes_{\mathrm{V}} Z_{Y}$ is a subspace of $C\left(B_{X^{*}}\right) \otimes_{\mathrm{V}}$ $C\left(B_{Y^{*}}\right)$ and thus isometrically isomorphic to a subspace of $C\left(B_{X^{*}} \times B_{Y^{*}}\right)$.

Now the injectivity of the Taylor complexification procedure (see [4, p. 14]) can be applied to get

$$
\begin{aligned}
\left(Z_{X} \otimes_{\vee} Z_{Y}\right)_{T} & \stackrel{1}{=} \text { a subspace of } C\left(B_{X^{*}} \times B_{Y^{*}}\right)_{T} \\
& \stackrel{1}{=} \text { a subspace of } C_{\mathbb{C}}\left(B_{X^{*}} \times B_{Y^{*}}\right) .
\end{aligned}
$$

Following all these isometries, we conclude that

$$
\begin{aligned}
B:\left(X \otimes_{\vee} Y\right)_{T} & \rightarrow C_{\mathbb{C}}\left(B_{X^{*}} \times B_{Y^{*}}\right), \\
\sum_{j=1}^{n}\left(e_{j} \otimes_{\vee} f_{j}+i\left(g_{j} \otimes_{\vee} h_{j}\right)\right) & \mapsto \sum_{j=1}^{n}\left(\widehat{e}_{j}(\cdot) \widehat{f}_{j}(\cdot \cdot)+i\left(\widehat{g}_{j}(\cdot) \widehat{h}_{j}(\cdot)\right)\right),
\end{aligned}
$$

is isometric.

On the other hand, $X \stackrel{1}{=} Z_{X}$ implies $X_{T} \stackrel{1}{=}\left(Z_{X}\right)_{T}$, same with $Y$, and now the injectivity of the Taylor procedure and then the injectivity of $\otimes_{V}$ yield

$$
\begin{aligned}
X_{T} \otimes_{\vee} Y_{T} & \stackrel{1}{=}\left(Z_{X}\right)_{T} \otimes_{\vee}\left(Z_{Y}\right)_{T} \\
& \stackrel{1}{=} \text { a subspace of } C\left(B_{X^{*}}\right)_{T} \otimes_{\vee} C\left(B_{Y^{*}}\right)_{T} \\
& \stackrel{1}{=} \text { a subspace of } C_{\mathbb{C}}\left(B_{X^{*}}\right) \otimes_{\vee} C_{\mathbb{C}}\left(B_{Y^{*}}\right) \\
& \stackrel{1}{=} \text { a subspace of } C_{\mathbb{C}}\left(B_{X^{*}} \times B_{Y^{*}}\right) .
\end{aligned}
$$

The canonical isometries combined in the above set of statements imply that the mapping

$$
\begin{aligned}
D: X_{T} \otimes_{\vee} Y_{T} & \rightarrow C_{\mathbb{C}}\left(B_{X^{*}} \times B_{Y^{*}}\right), \\
\sum_{j=1}^{n}\left(a_{j}+i b_{j}\right) \otimes\left(c_{j}+i d_{j}\right) & \mapsto \sum_{j=1}^{n}\left(\widehat{a}_{j}(\cdot)+i \widehat{b}_{j}(\cdot)\right)\left(\widehat{c}_{j}(\cdot \cdot)+i \widehat{d}_{j}(\cdot \cdot)\right),
\end{aligned}
$$

is isometric.

Let $u \in X_{T} \otimes_{\vee} Y_{T}=\left(X \otimes_{\vee} Y\right)_{T}$ be given. It is easy to verify that both $B$ and $D$ are complex-linear mappings, and that for any $x \otimes y$ in the vector space generating set $X \otimes Y$ we have $B(x \otimes y)=\widehat{x}(\cdot) \widehat{y}(\cdot \cdot)=D(x \otimes y)$; therefore, $B(u)=D(u)$.

Now because $B(u)=D(u)$ and both $B$ and $D$ are isometries, we conclude that $\|u\|_{X_{T} \otimes \vee} Y_{T}=\|D(u)\|=\|B(u)\|=\|u\|_{(X \otimes \vee Y)_{T}}$.

Clearly, by taking completions in the previous result, we get our main result on the complexification of the injective tensor product:

Theorem 2.3. Let $X, Y$ be real Banach spaces. The spaces $(X \stackrel{\vee}{\otimes} Y)_{T}$ and $X_{T} \stackrel{\vee}{\otimes} Y_{T}$ are isometrically isomorphic. 
3. The projective tensor product. A similar result holds when we consider the projective tensor norm. In this case, however, another complexification procedure should be used, as is indicated by the following. If we identify, as in [4], the complexification of $X$ with $X \otimes \ell_{2}^{2}$, the Taylor procedure arises when we view $x+i y=x \otimes e_{1}+y \otimes e_{2}$ as a member of $X \stackrel{\vee}{\otimes} \ell_{2}^{2}$, while the Bochnak norm is that of $x \otimes e_{1}+y \otimes e_{2} \in X \hat{\otimes} \ell_{2}^{2}$. So duality considerations hint that the Bochnak norm will do the trick.

Before we consider the complexification of the projective tensor product, we prove the following result (stated in [4] without proof). The result is not necessary for our treatment of the complexification of the projective tensor product, but it emphasises the dual relationship between these two complexification norms. We write $X_{B}$ for the Bochnak complexification of $X$.

Theorem 3.1. Let $X$ be a real Banach space. Then $\left(X^{*}\right)_{B} \stackrel{1}{=}\left(X_{T}\right)^{*}$ and $\left(X^{*}\right)_{T} \stackrel{1}{=}\left(X_{B}\right)^{*}$.

Proof. Recall that the Schatten dual norm $\alpha^{s}$ of a tensor norm $\alpha$ is defined on $X \otimes Y$ by the natural embedding

$$
X \otimes Y \subseteq\left(X^{*} \otimes_{\alpha} Y^{*}\right)^{*}
$$

It is known that in general

$$
X^{*} \otimes_{\left(\alpha^{s}\right)^{*}} Y^{*} \subseteq\left(X \otimes_{\alpha^{*}} Y\right)^{*} \quad \text { isometrically, }
$$

and that $\left(\alpha^{s}\right)^{*}=\alpha$ on $X \otimes_{\alpha} Y$ if $Y$ is finite-dimensional and $\alpha$ is accessible. Set $\alpha:=V$, which is known to be an accessible (even totally accessible) norm. Set $Y:=\ell_{2}^{2}$, so we have $Y^{*} \stackrel{1}{=} Y$. Hence $X^{*} \stackrel{\vee}{\otimes} Y^{*} \subseteq(X \hat{\otimes} Y)^{*}$, i.e. $\left(X^{*}\right)_{T} \subseteq\left(X_{B}\right)^{*}$ (isometrically). Now it is elementary to verify, by the finite-dimensionality of $Y$, that in fact equality holds in the last inclusion: $\left(X^{*}\right)_{T} \stackrel{1}{=}\left(X_{B}\right)^{*}$.

Since $\wedge$ is also an accessible tensor norm, the same argument shows that $\left(X^{*}\right)_{B} \stackrel{1}{=}\left(X_{T}\right)^{*}$.

Remark: An alternative argument, with $Y:=\ell_{2}^{2}$, is

$$
\begin{array}{rlrl}
X^{*} \hat{\otimes} Y^{*} & \stackrel{1}{=} \mathcal{N}\left(X, Y^{*}\right) & (Y \text { being finite-dimensional }) \\
& \stackrel{1}{=} \mathcal{I}\left(X, Y^{*}\right) \quad(Y \text { is reflexive }) \\
& \stackrel{1}{=}(X \stackrel{\vee}{\otimes} Y)^{*} . &
\end{array}
$$

Another reason why the Bochnak procedure is so compatible with the projective tensor product, is that it gives the "expected" complexification of $L^{1}(\mu)$-spaces (see [4]), hence of $\ell^{1}$-spaces. In other words, the Bochnak complexification of a real $\ell^{1}(\gamma)$-space is the complex $\ell^{1}(\gamma)$-space. Another essen- 
tial property of Bochnak's procedure is that it preserves quotients, i.e. for Banach spaces $X, Y$ the quotient space $(X / Y)_{B}$ is (isometrically isomorphic to) a quotient of $X_{B}$.

Recall the usual isometric surjection (valid for any Banach space $X$ ) $Q: \ell^{1}\left(B_{X}\right) \rightarrow X:\left(\lambda_{x}\right)_{x \in B_{x}} \mapsto \sum_{x \in B_{X}} \lambda_{x} x$. We will use the notation $\lambda^{x}$ for $\left(\lambda_{y}^{x}\right)_{y \in B_{X}}$ where

$$
\lambda_{y}^{x}:= \begin{cases}\|x\| & \text { if } y=x /\|x\| \\ 0 & \text { otherwise. }\end{cases}
$$

When there is another Banach space for which we consider such a surjection, we will use the symbol $\mu$ rather than $\lambda$ to avoid confusion.

Theorem 3.2. Let $X, Y$ be real Banach spaces. Then the Banach spaces $X_{B} \hat{\otimes} Y_{B}$ and $(X \hat{\otimes} Y)_{B}$ are isometrically isomorphic.

Proof. (Due to similarities with the proof of the previous result, we combine the steps in one theorem.) We first show that the mapping $T$ : $X_{B} \otimes Y_{B} \rightarrow(X \otimes Y)_{B}$ given by

$\sum_{k=1}^{n}\left(a_{k}+i b_{k}\right) \otimes\left(c_{k}+i d_{k}\right) \mapsto \sum_{k=1}^{n}\left(\left(a_{k} \otimes c_{k}-b_{k} \otimes d_{k}\right)+i\left(b_{k} \otimes c_{k}+a_{k} \otimes d_{k}\right)\right)$ is a linear bijective isometry.

It is clear that $T$ is complex-linear. Moreover, the set $B=\{x \otimes y$ : $x \in X, y \in Y\}$ has as complex span both $X_{B} \otimes Y_{B}$ and $(X \otimes Y)_{B}$; the former since $(a+i b) \otimes(c+i d)=a \otimes b+(i b) \otimes c+a \otimes(i d)+(i b) \otimes(i d)$ for any $a, b \in X$ and $c, d \in Y$, and the latter since $a \otimes b+i(c \otimes d)$ belongs to the span for any $a, b \in X$ and $c, d \in Y$. Now because $T(u)=u$ for any $u \in B$, we see at once that $T$ is surjective and injective.

To see that $T$ is isometric, consider the following diagram:

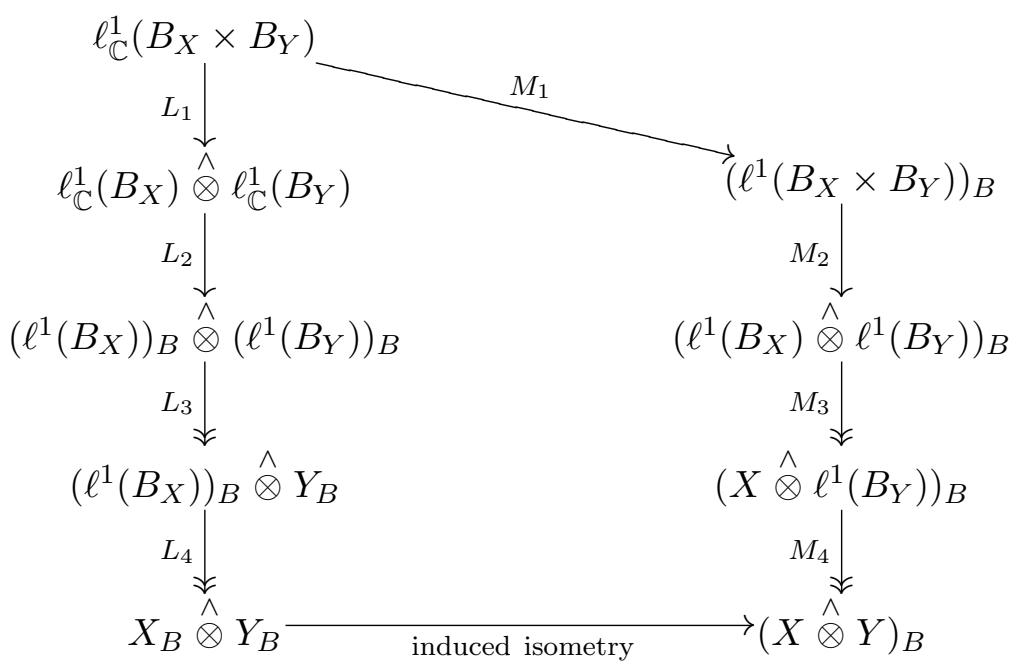


Here $L_{1}$ is the canonical isometry: It is a special case (with counting measure) of the isometric isomorphism between $L^{1}(\mu) \hat{\otimes} L^{1}(\nu)$ and $L^{1}(\mu \times \nu)$, which in turn can be deduced from the canonical isometry between $L^{1}(\mu) \hat{\otimes} Z$ and $L_{Z}^{1}(\mu)$ for any Banach space $Z$, shown for example in [2, Theorem 1.10].

The fact that $L_{2}$ is isometric is due to the above-mentioned result that the Bochnak complexification of any real $\ell^{1}$-space gives the complex $\ell^{1}$ space.

Now the fact that $Y$ is a quotient (under the usual quotient map) of $\ell^{1}\left(B_{Y}\right)$, together with the quotient property of the Bochnak procedure, implies that there is an isometric surjection $\left(\ell^{1}\left(B_{Y}\right)\right)_{B} \rightarrow Y_{B}$. Employing the quotient property of the projective tensor norm then yields an isometric surjection

$$
L_{3}:\left(\ell^{1}\left(B_{X}\right)\right)_{B} \hat{\otimes}\left(\ell^{1}\left(B_{Y}\right)\right)_{B} \rightarrow\left(\ell^{1}\left(B_{X}\right)\right)_{B} \hat{\otimes} Y_{B}
$$

Repeating the steps in the construction of $L_{3}$, but this time on the left hand side, yields an isometric surjection

$$
L_{4}:\left(\ell^{1}\left(B_{X}\right)\right)_{B} \hat{\otimes} Y_{B} \rightarrow X_{B} \hat{\otimes} Y_{B} .
$$

The mapping $M_{1}$ is isometric for the same reason as $L_{2}$ is. And $M_{2}$ is isometric for the same reason as $L_{1}$. The projective properties of the projective norm and of the Bochnak procedure give us the isometry $M_{3}$; and repetition, but this time on the right hand side, yields an isometric map $M_{4}$.

Let $S$ be the (complex) span of the set $\left\{\left(\lambda^{a}+i \lambda^{b}\right)\left(\mu^{c}+i \mu^{d}\right) \mid a, b \in X\right.$, $c, d \in Y\}$. Let $L$ be the map $L_{4} \circ L_{3} \circ L_{2} \circ L_{1}$, but with domain restricted to $S$. Thus $L: \ell_{\mathbb{C}}^{1}\left(B_{X} \times B_{Y}\right) \cap S \rightarrow X_{B} \otimes Y_{B}$ is the linearization of the map $\left(\lambda^{a}+i \lambda^{b}\right)\left(\mu^{c}+i \mu^{d}\right) \mapsto(a+i b) \otimes(c+i d)$. Similarly we let $M=$ $\left.\left(M_{4} \circ M_{3} \circ M_{2} \circ M_{1}\right)\right|_{S}$. Therefore $M$ is the linearization of the mapping $\ell_{\mathbb{C}}^{1}\left(B_{X} \times B_{Y}\right) \cap S \rightarrow(X \otimes Y)_{B}$ given by

$$
\left(\lambda^{a}+i \lambda^{b}\right)\left(\mu^{c}+i \mu^{d}\right) \mapsto(a \otimes c-b \otimes d)+i(b \otimes c+a \otimes d) .
$$

Now both $L$ and $M$ are compositions of isometric mappings, hence isometric themselves. This shows that $T$ is isometric, because for any member $v \in(X \otimes Y)_{B}$, there is $t \in \ell_{\mathbb{C}}^{1}\left(B_{X} \times B_{Y}\right)$ such that $L(t)=v$, thus if $T(u)=v$ then $\|u\|=\|L(t)\|=\|M(t)\|=\|T(u)\|$.

Thus $T: X_{B} \otimes Y_{B} \rightarrow(X \otimes Y)_{B}$ is a linear isometric bijection.

Since $X_{B} \otimes_{\wedge} Y_{B}$ and $\left(X \otimes_{\wedge} B\right)_{B}$ are isometrically isomorphic as normed vector spaces, their closures $X_{B} \hat{\otimes} Y_{B}$ and $(X \hat{\otimes} Y)_{B}$ are also isometrically isomorphic. 
We conclude with two remarks.

1. It is not known whether there are other real-tensor-norm/complextensor-norm pairs satisfying a relationship as in Theorems 2.3 and 3.2. In this remark we will discuss the case of the Hilbertian tensor norm, denoted by $H$. (See for example the book $[1,12.7]$ for the definition of $H$; this book uses the notation $w_{2}$ for $H$.)

Suppose one applies to the real Banach space $X$ the complexification procedure that results from using the $H$-norm on $X \otimes \ell_{2}^{2}$. Then, since the tensor norms $H$ and $\vee$ are equal on $X \otimes \ell_{2}^{2}$ for any Banach space $X$, this complexification is just the Taylor complexification procedure that was used in Section 2. The analogue of Theorems 2.3 and 3.2 would then be that

$$
(X \stackrel{H}{\otimes} Y)_{T} \stackrel{1}{=} X_{T} \stackrel{H}{\otimes} Y_{T}
$$

for all real Banach spaces $X$ and $Y$.

If $X$ and $Y$ are Hilbert spaces, it is then a consequence of Theorem 2.3 that $X_{T} \stackrel{H}{\otimes} Y_{T} \stackrel{1}{=}\left(X_{T} \stackrel{\vee}{\otimes} Y_{T}\right)$. However, $X_{T}$ and $Y_{T}$ are not Hilbert spaces. The author thinks that this last isometric isomorphism-and therefore the above-mentioned presumed analogue of Theorems 2.3 and 3.2 - cannot hold, because he believes that two Banach spaces $V$ and $W$, of which neither are Hilbert spaces, cannot satisfy the isometric isomorphism $V \stackrel{H}{\otimes} W \stackrel{1}{=} V \stackrel{\vee}{\otimes} W$.

2. The following general relationship between real and complex tensor norms holds if we consider isomorphic rather than isometric results. We say that tensor norms $\alpha_{1}$ and $\alpha_{2}$ are equivalent if there exist constants $c_{1}$ and $c_{2}$ such that for any $u \in X \otimes Y$, where $X$ and $Y$ are any Banach spaces over the same scalar field,

$$
\alpha_{1}(u) \leq c_{1} \alpha_{2}(u) \leq c_{2} \alpha_{1}(u) .
$$

Let $T_{r}$ denote the collection of equivalence classes of tensor norms in the real theory, and $T_{c}$ the collection of equivalence classes of tensor norms in the complex theory.

Then one can use the maximal properties of $\alpha$-integral operator ideals to show that there exists an injective mapping of $T_{r}$ into $T_{c}$. The fact that such a mapping exists was already claimed, without proof, in the Résumé, and the construction of such a mapping will be given in a forthcoming article.

\section{References}

[1] A. Defant and K. Floret, Tensor Norms and Operator Ideals, North-Holland, Amsterdam, 1993. 
[2] J. Diestel, J. Fourie, and J. Swart, The metric theory of tensor products (Grothendieck's résumé revisited). I. Tensor norms, Quaest. Math. 25 (2002), 37-72.

[3] A. Grothendieck, Résumé de la théorie métrique des produits tensoriels topologiques, Bol. Soc. Mat. São Paulo 8 (1953/1956), 1-79.

[4] G. A. Muñoz, Y. Sarantopoulos, and A. Tonge, Complexifications of real Banach spaces, polynomials and multilinear maps, Studia Math. 134 (1999), 1-33.

Department of Mathematics and Applied Mathematics

University of Pretoria

Pretoria 0002, South Africa

E-mail: gusti.vanzyl@up.ac.za

Received September 10, 2004

Revised version August 2, 2008 